\title{
$\sqrt{6}$

\section{La reutilización de aguas depuradas regeneradas a escala mundial: análisis y prospectivas}

\author{
Reuse of Purified Regenerated Water Worldwide: Analyzes and Projections
}

Daniel Prats-Rico

Universidad de Alicante. Alicante, España. Prats@ua.es

Resumen - El artículo presenta el estado de la reutilización de aguas residuales a escala mundial. Inicialmente se describen los parámetros que definen el mayor 0 menor déficit de agua, y se muestran las distintas regiones con déficit hídrico. Se revisan las principales causas que provocan el aumento en todo el mundo de esta problemática. Se incluye una breve reseña sobre sucesos históricos relacionados con el uso de aquas residuales, y se describen los usos posibles de las mismas. Se revisa el estado de la reutilización a escala mundial en los distintos países y regiones, según dos estudios realizados en 2008 y 2013. Finalmente se apuntan las principales razones para un continuo crecimiento de la reutilización de aquas residuales en el futuro, que crece paralelamente a la mejora en su tratamiento, identificando los países donde se espera mayor crecimiento. También se describe como las modernas tecnologías para el tratamiento de aguas residuales, permiten el uso directo o indirecto de las aguas regeneradas utilizándolas como aquas potables, describiendo los ejemplos más significativos.

Abstract - The article presents the situation of reused wastewater globally. At the outset we describe the parameters used to establish the higher or lower water deficit are, as well as the different regions where water supplies are under stress. The main causes of the worldwide increase of this problematic are reviewed. It includes a brief survey of historical events related to the use of wastewater and a description of the possible applications of this resource. Worldwide, the status of reused waters for different countries and regions is reviewed based on studies conducted in 2008 and 2013. The essay addresses the main reasons for the continued growth of reused wastewater in the future, whose expansion parallels advances in its treatment, and identifies the countries where a further increase of it is expected. It also describes how modern technologies for treating wastewater allow for its direct or indirect use for drinking purposes, indicating the most significant examples of this trend.

Palabras clave: agua, reutilización, aguas depuradas regeneradas, escala mundial
Keywords: water reuse, regenerated purified water, worldwide

Información Artículo: Recibido: 14 marzo 2016

Revisado: 12 septiembre 2016

Aceptado: 16 octubre 2016

(c) Universidad de J aén / Seminario Permanente Agua, Territorio y Medio Ambiente (CSIC) 


\section{LA NECESIDAD DE REUTILIZAR LAS AGUAS RESIDUALES}

Los problemas tecnológicos más importantes que afectan globalmente a la humanidad son el abastecimiento de energía y de agua en las cantidades suficientes para su bienestar y desarrollo.

En el caso de la energía los recursos disponibles proceden mayoritariamente de fuentes no renovables (y por tanto limitadas en el tiempo) y, además, su empleo provoca graves problemas ambientales (lluvia ácida, deterioro de la capa de ozono, cambio climático, contaminación radioactiva). Actualmente son claramente insuficientes para abastecer a todos los países, sobre todo, si se tiene en cuenta la demanda creciente de las economías emergentes.

En el caso del agua los recursos mayoritariamente utilizados hoy en día son renovables, a diferencia de los recursos energétiCos, y provienen del ciclo natural del agua. Los problemas surgen de su desigual distribución geográfica y temporal, lo que provoca desequilibrios entre los recursos disponibles y las necesidades de agua en múltiples áreas geográficas. En el mapa 1 se puede observar cómo se distribuye la precipitación media en las distintas regiones del mundo.

Se utilizan diversos criterios para medir el déficit hídrico de los territorios. Uno de ellos está relacionado con la cantidad de agua disponible por habitante y año, que considera distintos grados de escasez según se indica en la tabla 1.

En el mapa 2 se puede apreciar la situación de los distintos países teniendo en cuenta este criterio.

Un índice muy interesante para evaluar la situación de la disponibilidad de agua es la relación entre las necesidades totales de agua, para todos los usos, en una determinada cuenca, y los recursos naturales disponibles en dicha cuenca, medidos en las mismas unidades. Este criterio se denomina índice de estrés hídrico relativo (RWSI, Relative Water Stress Index), y se establece que:

- Si RWSI >0,4 se dan condiciones de estrés hídrico.

- Si RWSI <0,4 se dan condiciones de poco estrés 0 sin estrés.

Mapa 1. Ciclo global del agua. Precipitaciones medias

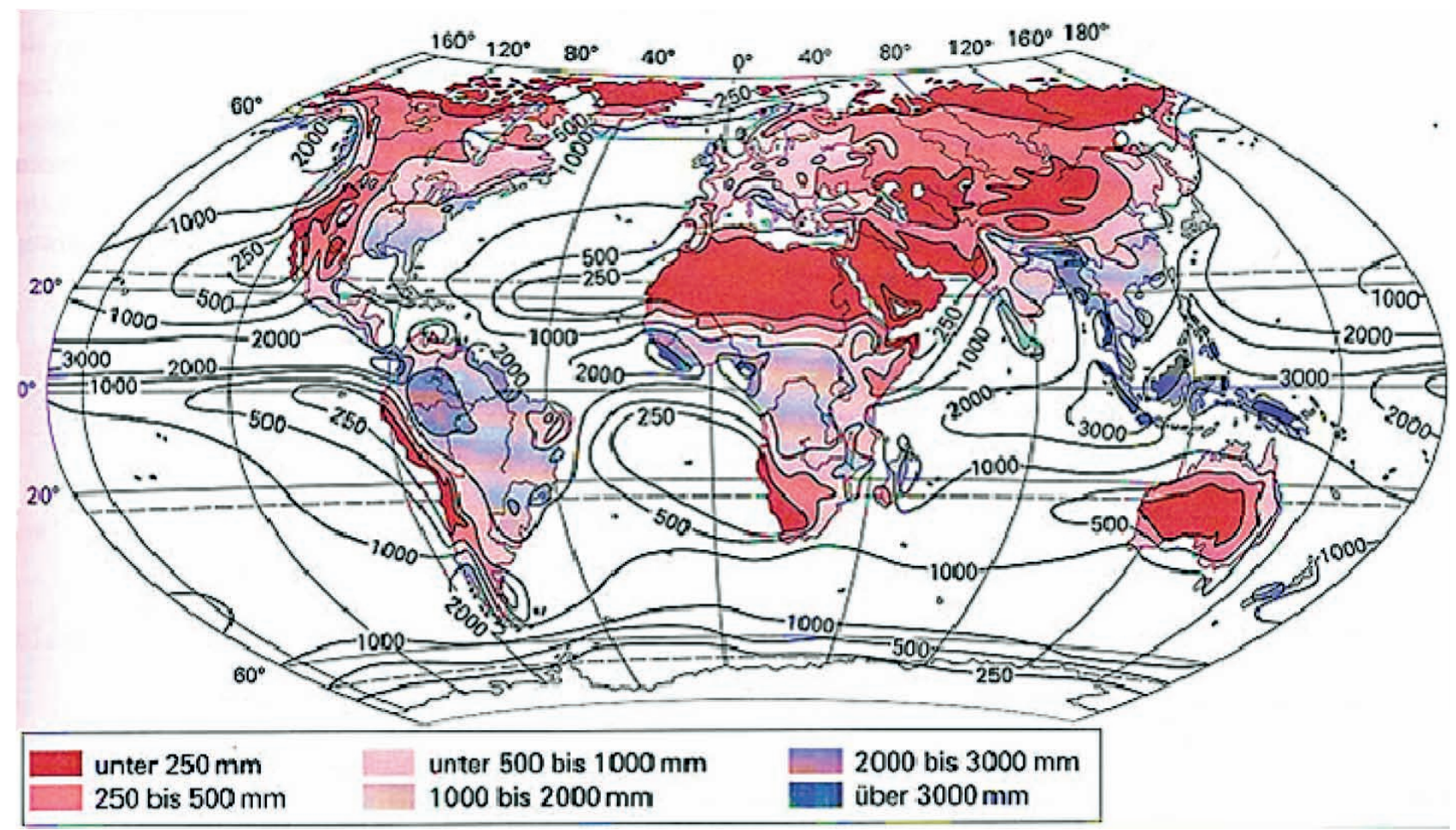

Fuente: Schertenleib \& Egli-Brož, 2011.

Tabla 1. Grados de escasez hídrica en función de la disponibilidad de agua

\begin{tabular}{|l|l|l|}
\hline $\begin{array}{c}\text { Disponibilidad de agua } \\
\mathrm{m}^{3} / \text { hab. día }\end{array}$ & \multicolumn{1}{|c|}{$\begin{array}{c}\text { Calificación de la situación } \\
\text { hídrica del territorio }\end{array}$} & \multicolumn{1}{c|}{ Consecuencias de la situación } \\
\hline $1.700-2.500$ & Vulnerabilidad & Puede haber zonas en los territorios considerados con algún tipo de restricción \\
\hline $1.000-1.700$ & Estrés & En determinadas épocas puede haber recursos limitados para al gunos usos del agua \\
\hline $500-1.000$ & Escasez & $\begin{array}{l}\text { Conflictos entre usos. Se debe acudir a recursos complementarios a los derivados del } \\
\text { ciclo del agua, como desalación y reutilización }\end{array}$ \\
\hline$<500$ & Escasez absoluta & Problemas sanitarios y un claro déficit para todas las aplicaciones \\
\hline
\end{tabular}




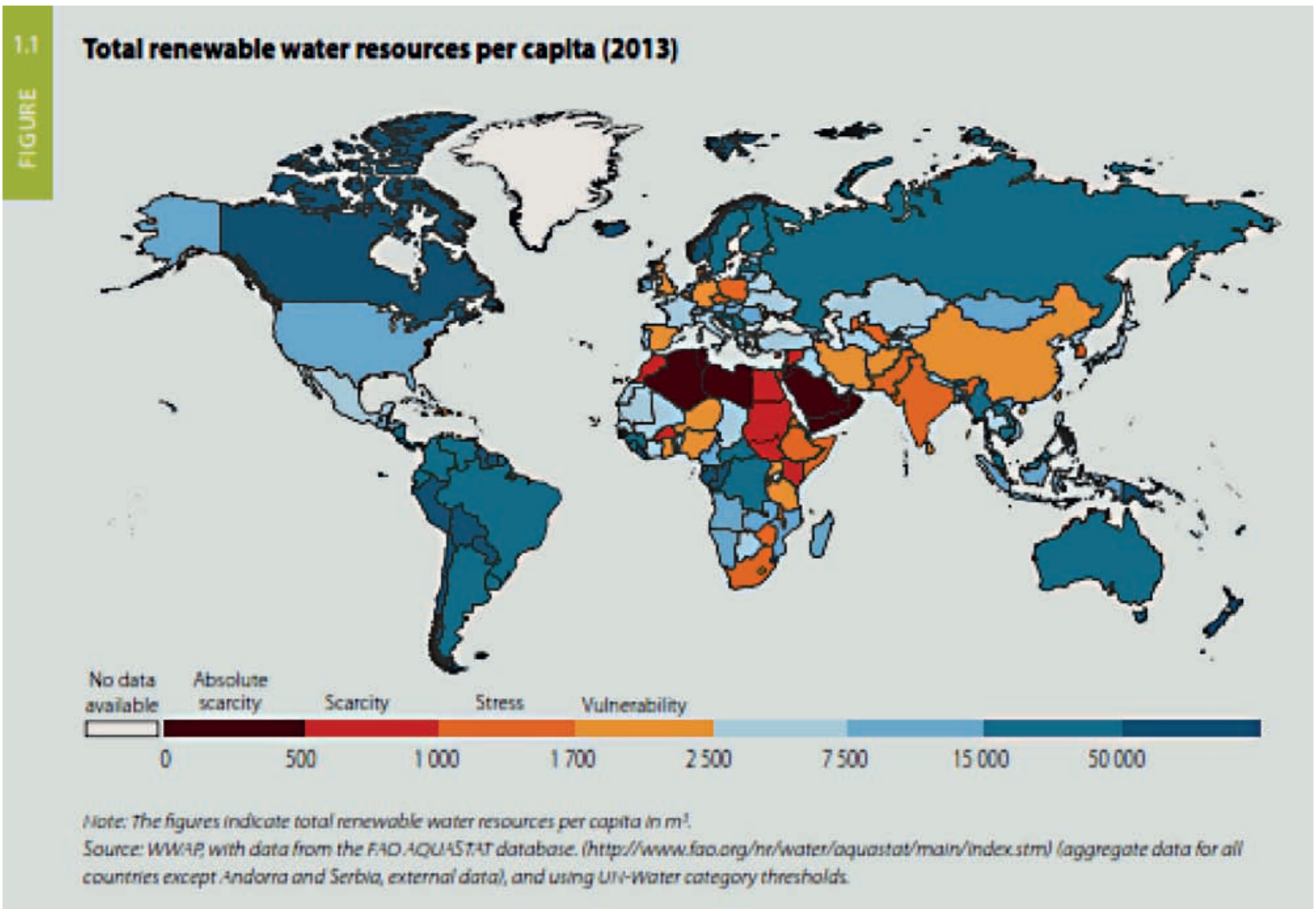

Fuente: World Water Development Report, 2015.

Mapa 3. Índice de estrés hídrico relativo, RWSI, a escala mundial

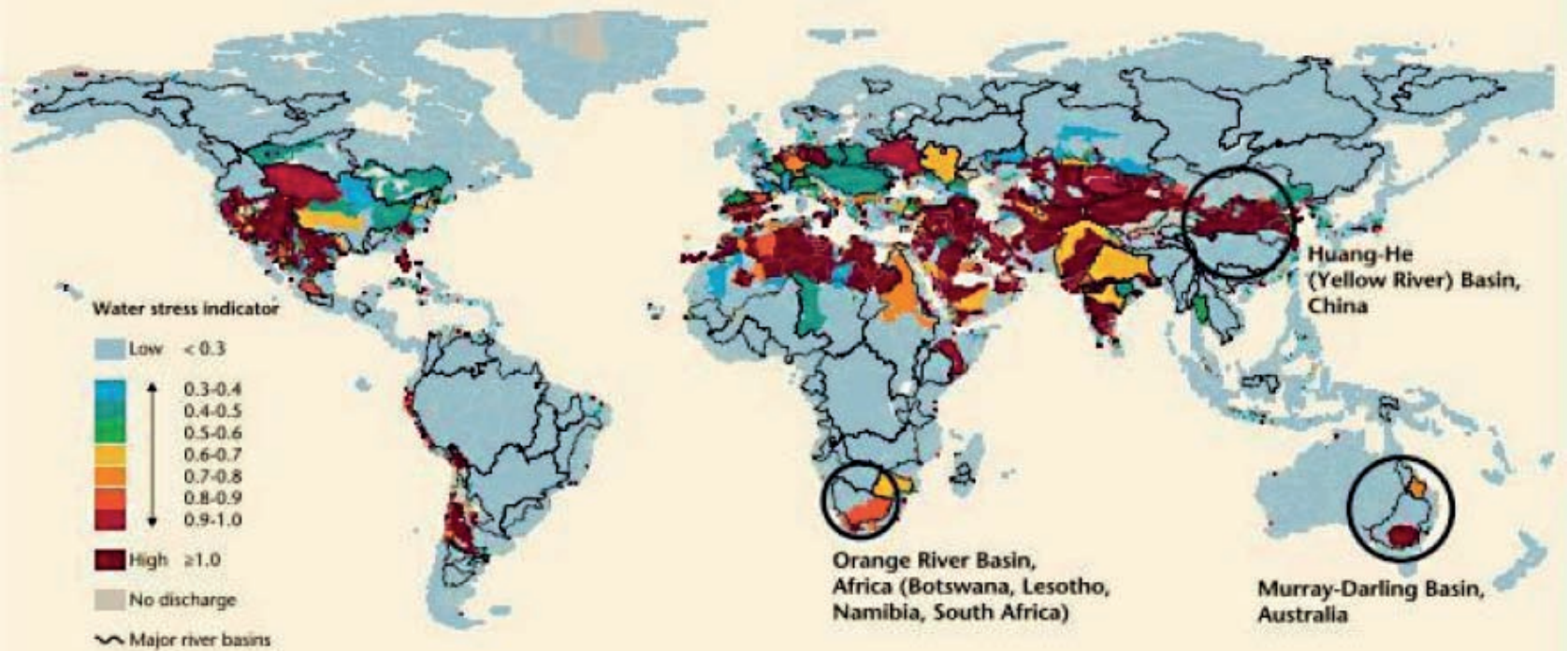

Source: Based on Smakhtin, Revenga, and Doll 2004.

Fuente: Fluet et al., 2009. 


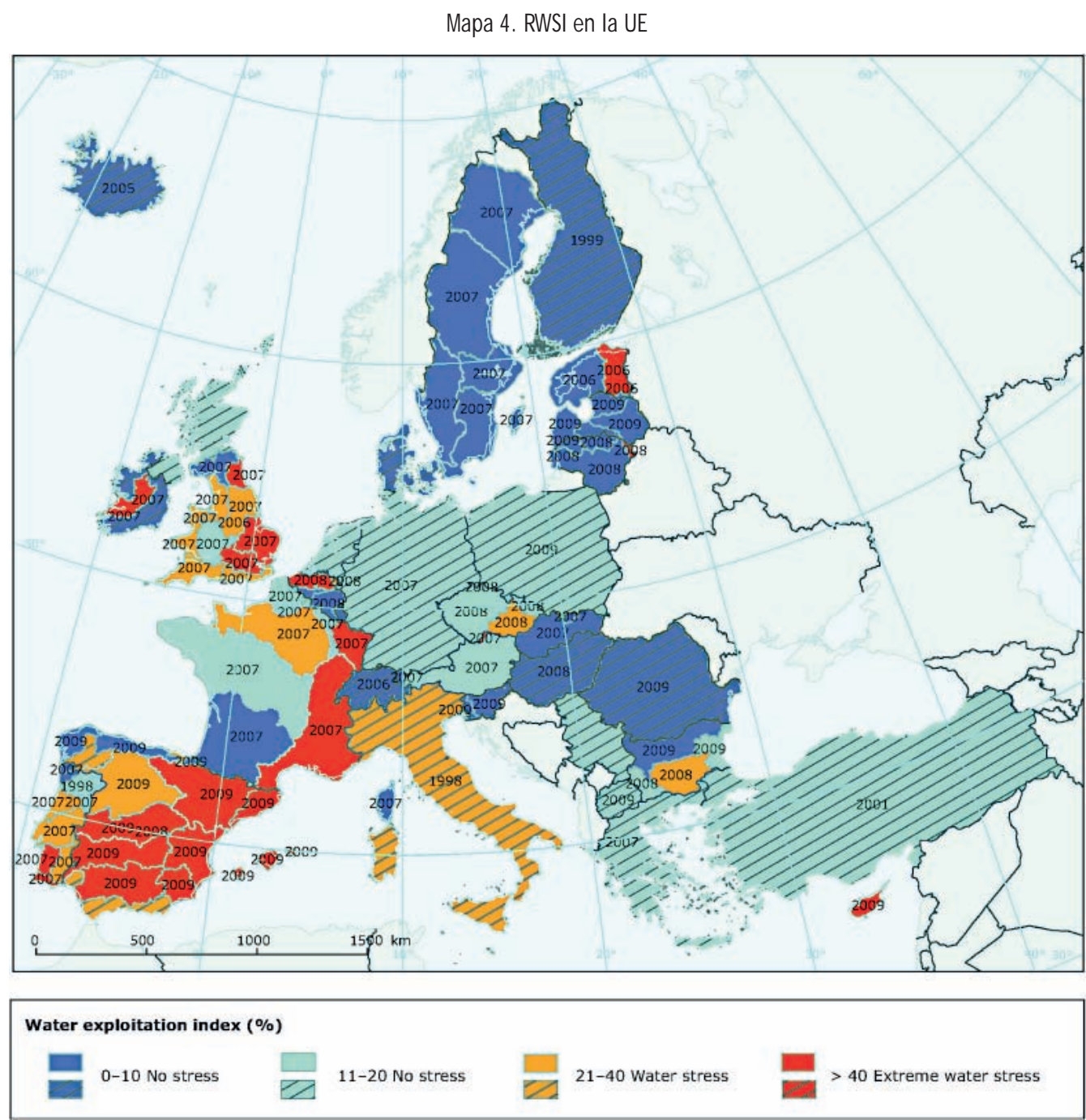

Fuente: Werner \& Collins, 2012.

Esto significa que si las necesidades de agua para todos los usos en una determinada zona, o sistema de explotación, superan el $40 \%$ de los recursos naturales disponibles, se considera que se dan condiciones de estrés. En el mapa 3 se muestra la distribución media a escala mundial del RWSI.

Como se puede observar, muchas zonas en el mundo se encuentran en la situación de elevados valores de RWSI.

A escala europea se puede observar con mayor detalle en el mapa 4 donde se aprecia que gran parte del territorio español está en situación de fuerte estrés hídrico.

Cuando la demanda de agua excede a las disponibilidades, se puede esperar la competencia creciente por el agua entre los usuarios. Con el aumento de la escasez de agua y la presión sobre los recursos hay una mayor probabilidad de conflictos y problemas para su administración, de que se contamine, de que se originen problemas de salud, así como del cierre de actividades económicas como áreas de regadío, etc.

Los desequilibrios que existen actualmente tienden a acentuarse a escala mundial. Se pueden indicar hasta siete causas importantes de carácter general:
- El crecimiento de la población y su mayor longevidad.

- El creciente desarrollo de países muy poblados. Mayores demandas de seguridad alimentaria y bienestar económico.

- Los grandes cambios demográficos con desplazamiento de la población del entorno rural al urbano.

- La contaminación de las aguas.

- Los periodos de sequía.

- La progresiva deforestación y desertización en muchas regiones.

- Las consecuencias imprevisibles del cambio climático.

Todos estos factores son importantes e influyen en la progresiva escasez de recursos hídricos de calidad a escala mundial. El efecto del crecimiento de la población es fácilmente cuantificable, como se puede apreciar en el gráfico 1, donde se representa la evolución de la disponibilidad de agua renovable por habitante y año, con una proyección hasta 2050. La línea azul representa la disponibilidad total y la línea verde la disponibilidad real, una vez restados los caudales de los grandes ríos que van al mar, las escorrentías de ramblas que desembocan en el mar, los recursos en zonas no habitadas, etc. 
Gráfico 1. Evolución del número de habitantes en el mundo y de la disponibilidad media de agua

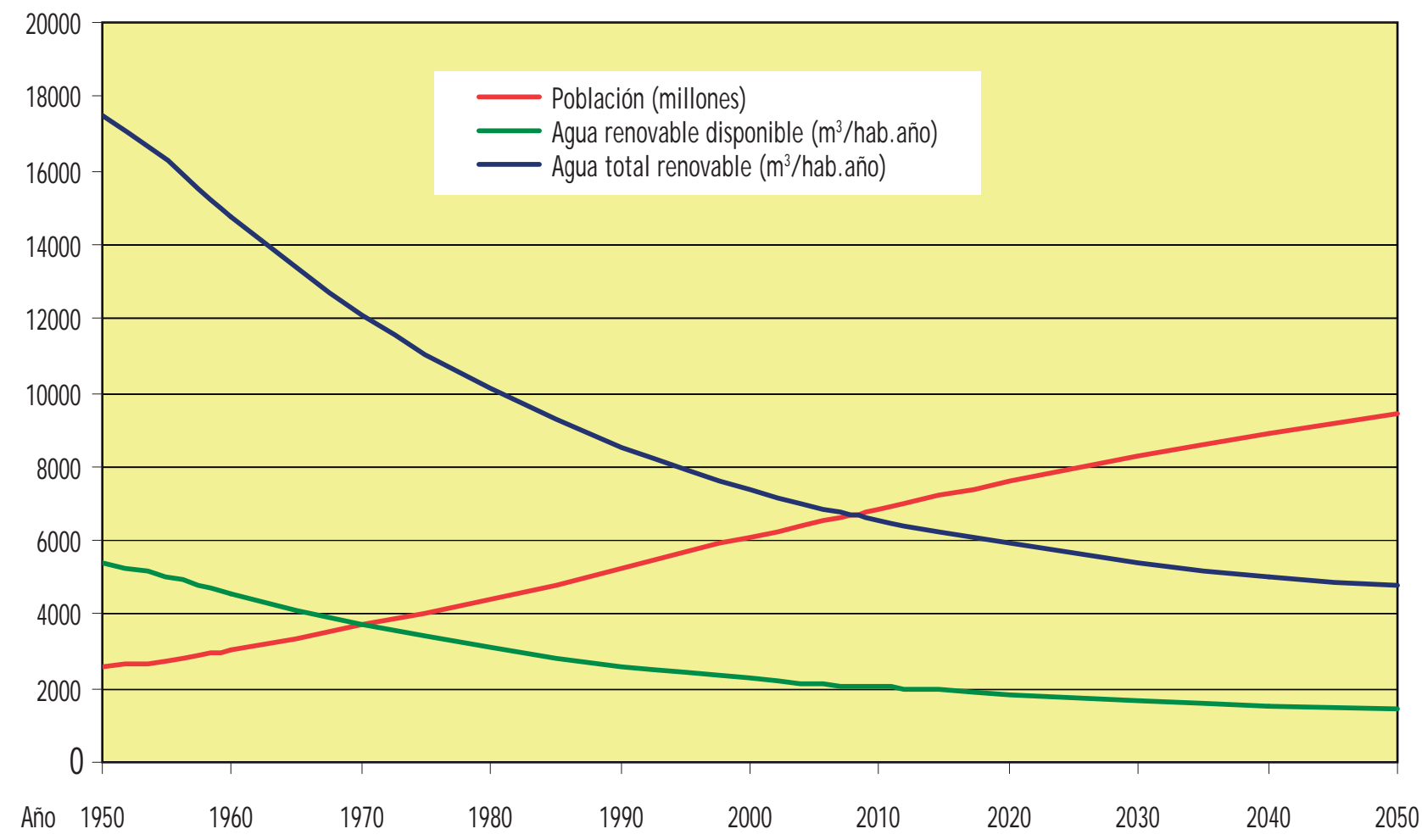

Fuente: elaboración propia.

En 2016, con una población mundial de 7.400 millones de habitantes, el caudal de agua renovable disponible es inferior a $2.000 \mathrm{~m}^{3} / \mathrm{hab}$. año. Si se tiene en cuenta que la propia ONU estima en $1.500 \mathrm{~m}^{3} /$ hab. año el caudal razonable para cubrir todas las necesidades, se deduce la grave situación actual y futura para muchos países y regiones, dados los desequilibrios geográficos y temporales de la distribución de agua y las desigualdades en las disponibilidades técnicas y económicas para su aprovechamiento.

Para minimizar o resolver la problemática del agua en las regiones con déficit hídrico caben dos tipos de actuaciones, gestionar mejor y obtener nuevos recursos. La reutilización planificada del agua es una forma evidente de obtener nuevos recursos.

El notable desarrollo alcanzado por la reutilización planificada del agua, especialmente en países con recursos hídricos insuficientes, se ha debido tanto a la necesidad de ampliar los recursos de agua para atender una demanda en permanente expansión, como a la exigencia de mejorar las formas de gestión de los vertidos de aguas depuradas. Las aguas regeneradas deben considerarse como un recurso no convencional, cuya gestión debe incluirse en una planificación integral de los recursos hídricos, que tenga en cuenta los aspectos económicos, sociales y medioambientales. La mejora de la calidad de los efluentes es el elemento clave en el aprovechamiento y la gestión del agua. Así, el agua regenerada puede sustituir usos en los que se esté utilizando agua de gran calidad, como determinados usos urbanos o industriales, y liberar estos caudales para otros usos más exigentes.
Cabe citar, al menos, las siguientes ventajas de la reutilización directa planificada:

- El aumento de la disponibilidad de agua en zonas sin reutilización.

- El uso integrado y sostenible de los recursos hídricos.

- Mantener el agua potable para beber y el agua regenerada para uso no potable.

- Reducir la extracción de agua superficial y subterránea.

- Contribuir a la reducción del consumo de energía en comparación con el uso de los recursos de aguas subterráneas profundas, aguas trasvasadas 0 aguas desaladas.

- Reducir las cargas de contaminantes a las aguas receptoras.

- Aumentar la producción agrícola.

- Reducir el uso de fertilizantes.

- Al ser un recurso estable, permite mayor fiabilidad en su disponibilidad.

- Una mayor protección del medio ambiente mediante la restauración de arroyos, humedales y lagunas.

- Aumentar el empleo y la economía local (por ejemplo, turismo, agricultura).

Ahora bien, hay que considerar de igual modo una serie de inconvenientes que pueden limitar, o en algunos casos imposibilitar, la alternativa de la reutilización. Entre los inconvenientes se pueden indicar:

- La necesaria calidad asociada al uso, lo que implica un tratamiento apropiado. 
Figura 1. Calidad del agua en función de su tratamiento

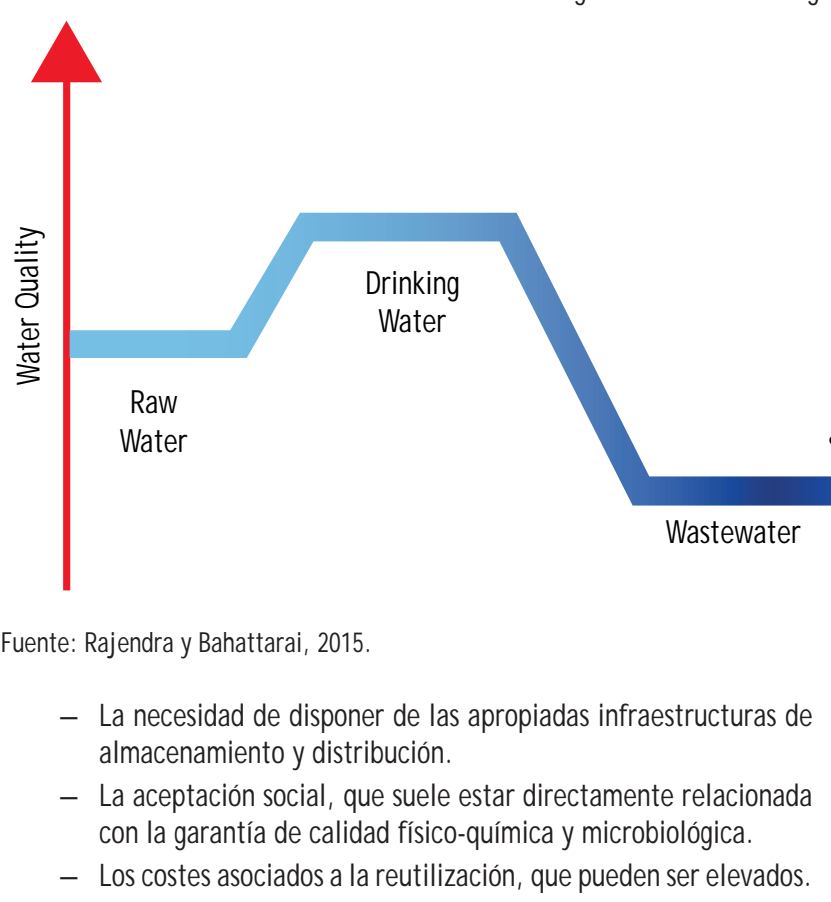

\section{Reseña histórica de LA ReUtiLIZACIÓN A ESCALA MUNDIAL}

El uso de aguas residuales para regadío se remonta a la antigüedad. En la civilización minoica, que surgió en la isla de Creta en la Edad del Cobre, año 3.000 a. C., ya se usaba agua residual para la agricultura. Algunos hechos significativos relacionados con la evolución de la demanda de aguas residuales y su reutilización ${ }^{1}$ son:

- Año 97. La ciudad de Roma nombra un Comisionado para el suministro de agua, Sexus J ulius Frontinus.

- Año 1500. En Alemania se emplean depuradoras para el tratamiento de aguas residuales.

- Año 1700. En Reino Unido se emplean depuradoras para el tratamiento de aguas residuales.

- 1800-1880. Se establece el uso de alcantarillado en París (1880), Londres (1815) y Boston (1833).

- 1850-1875. Se vincula una larga epidemia de cólera en Londres con la contaminación de los pozos de suministro de agua.

- 1850-1875. En Inglaterra Budd desarrolla una teoría para la prevención de la fiebre tifoidea.

- 1850-1875. En Alemania Koch demuestra la conexión del ántrax con la etiología bacteriana.

- 1875-1900. En Francia Pasteur demuestra la contaminación bacteriana del agua. En Alemania Down propugna el uso de hipoclorito sódico como desinfectante.

- 1890. En Ciudad de México se construyen canales de drenaje para tomar aguas residuales destinadas al riego de una importante zona agrícola al norte de la ciudad. Aguas residuales no tratadas o tratadas mínimamente llegan desde Ciudad de Méxi-

\footnotetext{
1 Levine et al., 2010.
}

co al Valle de México, donde se utilizan para el riego de 90.000 ha de tierras agrícolas, incluidas verduras.

- 1906. En J ersey City, NJ, USA, se empiezan a clorar las aguas suministradas.

- 1906. En el Boletín Mensual del Consejo de Salud del Estado de California, USA, se hace la referencia más temprana a la calidad del agua desde el punto de vista de la salud pública, en febrero de 1906, relativa al tanque séptico de la ciudad de Oxnard: "¿Por qué no utilizarla para el riego y mantener las valiosas propiedades fertilizantes en solución, y al mismo tiempo purificar completamente el agua? La combinación de la fosa séptica y el riego parece el sistema más racional, barato y eficaz para este estado".

- 1913-1914. El proceso de lodos activados fue demostrado por Ardern y Lockett en Inglaterra, y se desarrolló en la Estación Experimental Lawrence en Massachusetts.

- 1922. Se construye una planta de tratamiento de aguas residuales industriales en Mikawajima, Tokio, J apón.

- 1926. En el Parque Nacional del Gran Cañón, USA, el agua residual tratada se utiliza por primera vez en un sistema dual de agua, para la cisterna del inodoro, la aspersión del césped, el agua de refrigeración y el agua de alimentación de calderas.

- 1929. La ciudad de Pomona, en California, inicia un proyecto para regar jardines y céspedes con agua regenerada.

- 1968. En Namibia comienza la reutilización directa del agua residual como agua potable. Con al gunas modificaciones en el sistema de tratamiento, esta actividad continúa en la actualidad.

Se puede afirmar que a partir de 1960 y hasta la actualidad se han desarrollado múltiples avances tecnológicos en el tratamiento de las aguas naturales y de las aguas residuales que propician alcanzar aguas regeneradas de calidad apropiada para los distintos usos posibles. De hecho la calidad de las aguas regeneradas puede ser superior al de las aguas naturales, tal como se esquematiza en la figura 1.

\section{Posibles usos del agua residual}

Los usos posibles del agua residual a escala mundial pueden ser muy variados: 
- Municipales. Riego de parques públicos, instalaciones deportivas, jardines privados, bordes de carreteras; limpieza de las calles; sistemas de protección contra incendios; lavado de vehículos; cisterna del inodoro, uso en acondicionadores de aire, baldeo de calles.

- Agrícolas. Riego de cultivos de alimentos no procesados comercialmente o procesados comercialmente, pastos para los animales de ordeño, forraje, fibra, cultivos de semillas, flores ornamentales, huertos, cultivo hidropónico, acuicultura, invernaderos, viticultura.

- Industriales. Agua de refrigeración, torres de refrigeración, agua de lavado, lavado de áridos, fabricación de hormigón, compactación del suelo, control del polvo.

- Recreativos. Riego de campos de golf, embalses recreativos con 0 sin acceso público (por ejemplo, para pesca, navegación, baño), embalses ornamentales sin acceso público, nieve artificial.

- Ambientales. Recarga de acuíferos, humedales, pantanos; caudales ecológicos, hábitat de vida silvestre, silvicultura.

- Uso como potable. Recarga de acuíferos para agua potable, aumento de los suministros de agua potable de superficie, tratamiento hasta la calidad de agua potable.

Se reutiliza agua residual con distintos grados de depuración. También se usa sin tratar, sobre todo en países poco desarrollados de América Latina, Asia y África subsahariana.

\section{Gráfico 2. Usos del agua reutilizada a escala mundial}

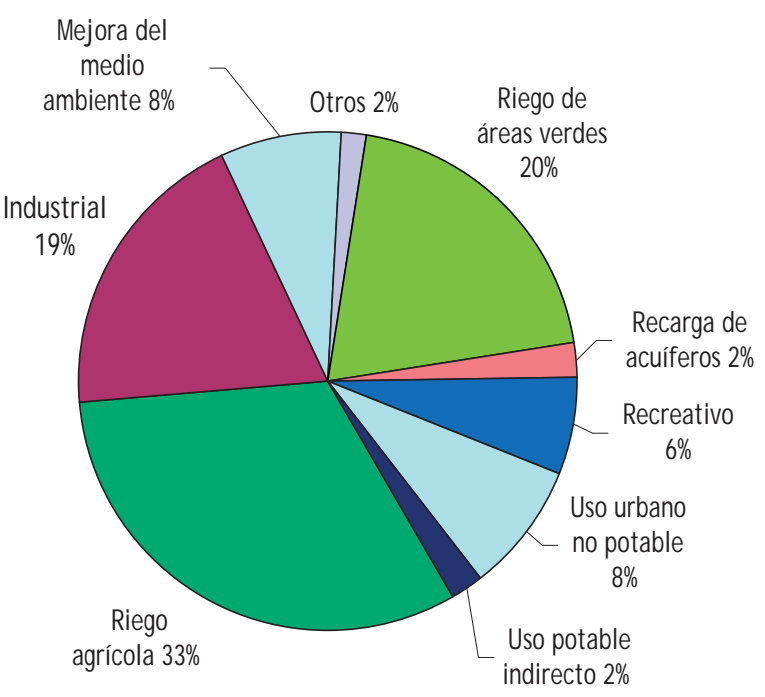

Fuente: adaptado de Intelligence, 2009

En países desarrollados y para aguas con tratamiento secundario o superior, se estima que los usos a escala mundial se distribuyen como se indica en el gráfico 2.

Como se puede observar, los usos principales van orientados al regadío y la industria, siendo muy pequeño el porcentaje de uso urbano. Este hecho parece relacionado con los usos de las aguas dulces, ya que la demanda y consumo de agua para uso agrícola es, con gran diferencia, el sector que más agua demanda. Ahora bien, existen razones importantes para prever un aumento en los usos urbanos y en los usos industriales de calidad:
- Los usos en las ciudades e industrias próximas a las depuradoras disminuyen el coste de infraestructuras de transporte y los gastos de bombeo.

- Los usos urbanos e industriales pueden ser menos estacionales que los agrícolas, lo que disminuye el coste de almacenamiento.

- Con la escasez de agua se acentúa la competencia entre usos, prevaleciendo aquellos que tienen mayor valor añadido y que permiten a los usuarios cubrir el coste del tratamiento y gestión del agua depurada, o estar menos subvencionados.

Las razones comentadas llevan a la necesidad de obtener efluentes de alta calidad para posibilitar usos urbanos e industriales, así como la recarga de acuíferos para su recuperación y para posibilitar el uso indirecto del agua regenerada como potable. Por ello es previsible un fuerte crecimiento en tratamientos terciarios convencionales y avanzados para las aguas residuales.

\section{SituACión de La REUTILIZACIÓN A ESCALA MUNDIAL}

Jiménez y Asano realizaron en 2008 un estudio estadístico encontrando que unos cincuenta $\mathrm{Mm}^{3} /$ día de agua residual se reutilizaban en todo el mundo. La mayor parte de este volumen se emplea sin tratar, principalmente en China (14,1 Mm³/ día) y México (13,6 Mm³/ día). Los principales países que según el estudio citado reutilizan agua tratada son USA (7,7 $\mathrm{Mm}^{3} /$ día), Arabia Saudí $\left(1,9 \mathrm{Mm}^{3} /\right.$ día), Egipto $\left(1,8 \mathrm{Mm}^{3} /\right.$ día), Israel $\left(1,0 \mathrm{Mm}^{3} /\right.$ día $)$ Siria (1,0 $\mathrm{Mm}^{3} /$ día) y España $\left(0,8 \mathrm{Mm}^{3} /\right.$ día).

En una revisión más reciente sobre los datos disponibles a escala regional, nacional y global realizada por Sato et al. en 2013, se deduce que de 181 países estudiados en 55 de ellos hay información disponible sobre generación, tratamiento y utilización de aguas residuales, en 69 hay información sobre generación y tratamiento y en 57 no hay información. No obstante, a pesar de que la información no es completa y además no está en algunos casos actualizada, se pueden extraer conclusiones interesantes sobre la situación de la reutilización a escala mundial. El resumen de la situación en distintas regiones del mundo es el siguiente:

Norteamérica (excluyendo México). Cada año se generan 85 $\mathrm{km}^{3}$ de aguas residuales, de los que se tratan $61 \mathrm{~km}^{3}(75 \%$ y se reutilizan $2,3 \mathrm{~km}^{3}(3,8 \%)$. Un porcentaje elevado de agua reutilizada (46\% en California, 44\% en Florida) se emplea en regadío, estimándose en 15.000 ha las zonas regadas en USA.

América Latina. Solo hay información completa para 9 de los 32 países de América Latina. De los países con información se puede calcular una generación de aguas residuales de $29 \mathrm{~km}^{3}$, de los que se tratan $5 \mathrm{~km}^{3}(18 \%)$ y se reutilizan $0,6 \mathrm{~km}^{3}(2 \%)$. En México entre de 70.000 y 190.000 ha se riegan con aguas residuales tratadas y no tratadas, respectivamente. En Perú 1.350 y 9.346 ha se riegan con aguas residuales tratadas y no tratadas. En Argentina y Chile las áreas regadas con aguas residuales tratadas son similares o más grandes que las áreas regadas con aguas residuales sin tratar. Se puede deducir que la escasez de agua no es el principal impulsor del uso de aguas residuales en la mayor parte de América Latina. Los agricultores usan aguas residuales porque proporcionan una fuente de bajo costo de nutrientes para los cultivos. 
Federación de Rusia y Estados Independientes de la Unión Soviética. Hay información completa de 8 países y parcial de 7. Cada año se generan $28 \mathrm{~km}^{3}$ de aguas residuales, de los que se tratan $20 \mathrm{~km}^{3}\left(71 \%\right.$ y se reutiliza $1,0 \mathrm{~km}^{3}(3,6 \%)$.

Oriente Medio y Norte de África. Con la excepción de Argelia e Irak, está disponible información completa sobre la generación de aguas residuales, tratamiento y uso en todos los países de la región. Cada año se generan $22,3 \mathrm{~km}^{3}$ de aguas residuales, de los que se tratan $11,4 \mathrm{~km}^{3}(51 \%)$ y se reutilizan $3,7 \mathrm{~km}^{3}(17 \%$. Como se puede apreciar hay un elevado porcentaje de uso de aguas residuales tratadas, lo que es esencial en esta región por su escasez de agua. Algunos países de la región tienen previsto aumentar el uso de aguas residuales tratadas. Por ej emplo, Arabia Saudita tiene la intención de aumentar el uso de las aguas residuales hasta el $65 \%$ en 2016 , mientras que Israel utiliza ya el $70 \%$ de las aguas residuales en el sector doméstico. Uso de aguas residuales representa alrededor del $10 \%$ del suministro nacional de agua israelí y casi el 20\%del suministro de agua para el riego.

África Sub-sahariana. De los 48 países de esta región únicamente hay información completa de tres de ellos, y de otros 13 sólo información parcial, por lo que para esta región la información es muy limitada. De los pocos países con información se puede calcular una generación de aguas residuales de $3,7 \mathrm{~km}^{3}$, mayoritariamente de Sudáfrica $(3,2)$, de los que se tratan 3,3 $\mathrm{km}^{3}(89 \%)$, mayoritariamente de Sudáfrica $(3,2)$ y se reutilizan $0,06 \mathrm{~km}^{3}(2 \%)$. En la mayoría de los casos, las aguas residuales que se utilizan para la agricultura están contaminadas y suelen ser sin tratar.

Oceanía. La información completa sobre los tres aspectos de aguas residuales está disponible sólo para Australia. El volumen de agua residual tratada está disponible para Nueva Zelanda. No hay información con respecto a Fiji y las Islas Salomón. Hay una generación de aguas residuales de $2,1 \mathrm{~km}^{3}$, en Australia, y se tra$\tan 2,3 \mathrm{~km}^{3}$, en Australia y Nueva Zelanda, reutilizándose 0,35 $\mathrm{km}^{3}$ en Australia. Alrededor del $45 \%$ de los 450 proyectos de uso de aguas residuales en Oceanía son para la agricultura. En Australia, los 0,35 $\mathrm{km}^{3}$ de aguas residuales tratadas que se utilizan anualmente representan el $19 \%$ de las aguas residuales tratadas en el país y alrededor del $4 \%$ del suministro total de agua. La agricultura es el sector mayoritario del uso de aguas residuales en Australia, donde unas veinte mil ha se riegan con aguas residuales tratadas. En Nueva Zelanda, las aguas residuales se utilizan para el riego de campos de golf y para aplicaciones industriales, pero los volúmenes implicados son pequeños.

Asia. La información completa está disponible solo en cinco países, mientras que en otros 14 hay datos parciales. De la información disponible se puede calcular una generación de aguas residuales de $132 \mathrm{~km}^{3}$, de los que se tratan $42 \mathrm{~km}^{3}$ (32\%) y se reutilizan $14 \mathrm{~km}^{3}(11 \%)$. Se estima que 1,3 millones de hectáreas se riegan con aguas residuales en China. El uso de aguas residuales no tratadas es habitual en países como Vietnam, Pakistán o la India. Japón ha adoptado una estrategia integral para el uso de aguas residuales tratadas. En 2009, se utilizaron $0,2 \mathrm{~km}^{3}$ de aguas residuales tratadas en este país. Más de la mitad se utilizó con fines ambientales, tales como el riego de jardines (27\%), recrea- ción (2\%) y el mantenimiento de los caudales en ríos (29\%). En Japón el uso de aguas residuales en la agricultura y la industria representa solamente el $7 \%$ y $1 \%$ de las aguas residuales tratadas, respectivamente. Además, más del $3 \%$ del agua residual tratada se utiliza para descargas en baños. Esta estrategia de uso de aguas residuales en J apón es algo único, ya que se centra en los usos de agua urbanos, en lugar de proporcionar agua principalmente para usos agrícolas.

Europa. En la revisión de Sato ${ }^{2}$ se dispone de información completa sobre la generación de aguas residuales, tratamiento y uso para diez países de Europa, e información parcial del resto, 31 países. Los datos disponibles conducen a una generación de aguas residuales de $52 \mathrm{~km}^{3}$, de los que se tratan $35 \mathrm{~km}^{3}$ (67\%) y se reutilizan $1,4 \mathrm{~km}^{3}(2,7 \%)$. El uso de aguas residuales está condicionado por la geografía. En el sur de Europa las aguas residuales regeneradas se utilizan principalmente para el riego agrícola (44\% de los proyectos de aguas residuales) y aplicaciones urbanas 0 ambientales (37\% de los proyectos). En el norte de Europa, las aguas residuales se utilizan principalmente para las aplicaciones ambientales (51\% de los proyectos) y la industria, que representa el $33 \%$ de los proyectos. Respecto a España, el $71 \%$ del volumen de aguas residuales se utiliza para el riego, 17\%para aplicaciones ambientales, 7\% para la recreación, 4\% para la reutilización urbana y el 0,3\% para fines industriales.

\section{El futuro de la Reutilización de aguas Residuales}

En la actualidad la reutilización a escala mundial de aguas residuales municipales está en clara expansión.

En la publicación de Global Water Intelligence ${ }^{3}$ se realiza un análisis exhaustivo sobre las previsiones de crecimiento. Se apuntan cuatro razones principales para este crecimiento de la reutilización:

1. La escasez de agua derivada del creciente aumento económico y demográfico. Cientos de millones de personas en muchas partes del mundo dependen actualmente de recursos subterráneos sobreexplotados. El cambio climático plantea la posibilidad de que las sequías prolongadas se conviertan en fenómenos recurrentes. También llama la atención el consumo de energía en el sector del agua (en California, por ejemplo, el $20 \%$ del consumo de energía total del estado es en el transporte y tratamiento de agua) y los requisitos adicionales de agua de los biocarburantes (un litro de bioetanol podría requerir hasta 2.100 litros de agua para hacer crecer la materia prima y para el proceso de transformación).

2. Las preocupaciones medioambientales están pasando a primer plano en la crisis del agua. Históricamente, la primera solución a la escasez de agua ha sido la de construir grandes presas y la transferencia de agua. Estos enfoques están ahora ampliamente considerados como perjudiciales para el medio ambiente. Por otra parte, la desalinización de agua de mar requiere energía, y la toma de agua y evacuación de salmueras pueden tener un impacto sobre el medio ambiente marino si no son adecuadamente

2 Sato et al., 2013.

3 Intelligence, 2009. 


\section{Daniel Prats-Rico}

Gráfico 3. Evolución prevista de la capacidad instalada de los distintos grados de tratamiento de las aguas residuales

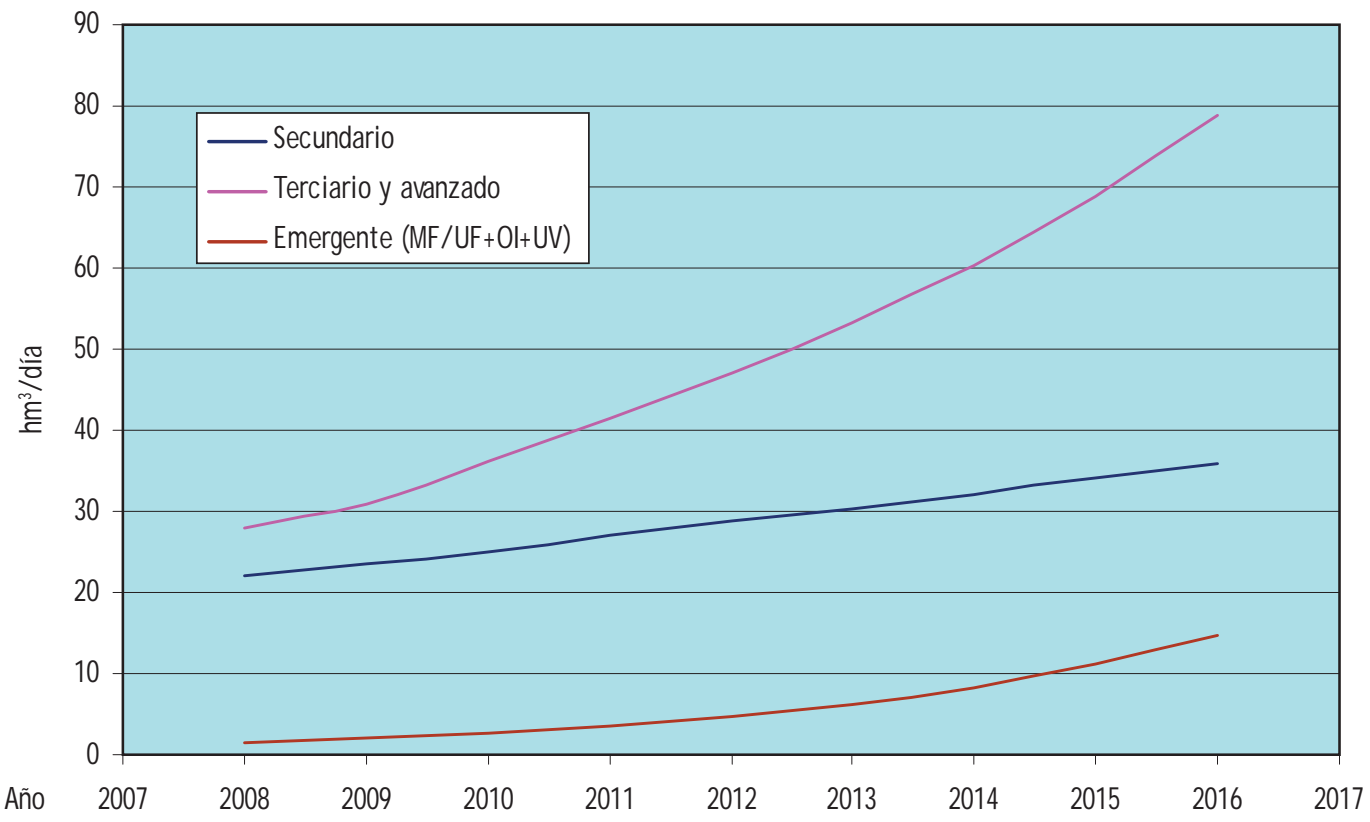

Fuente: Intelligence, 2009.

mitigadas. La reutilización del agua utiliza menos energía que la desalinización, y su aplicación en general es beneficiosa para el medio ambiente. Esta es una razón clave por la que actualmente se da prioridad a esta solución para paliar la escasez de agua por los políticos de todo el mundo.

3. El valor económico que representa la reutilización del agua. Sin reutilización, el tratamiento de aguas residuales tiene un valor ambiental, pero sin ningún valor financiero. La reutilización del agua, añade un nuevo valor a la propuesta. La inversión en infraestructura de aguas residuales se motiva no solo por la necesidad de los servicios públicos para cumplir con las regulaciones del medio ambiente, sino porque la reutilización del agua crea la posibilidad de vender el producto final. Incluso cuando el agua reciclada no se vende a un precio de mercado, el gasto necesario para mejorar el tratamiento de aguas residuales para la reutilización del agua, puede ahorrar mucho dinero que habría que gastar en otros proyectos de suministro de agua. La actual crisis financiera ha reforzado los argumentos económicos para la reutilización del agua. El sector del agua es un componente importante y clave del paisaje eco-industrial a escala mundial. El mercado mundial del agua está creciendo rápidamente y se estima que puede llegar a un billón de euros en 2020. Por otra parte, el potencial de innovación en el campo de la gestión del agua podría contribuir de manera significativa a la creación de empleo y la competitividad. Por ejemplo, en Europa un aumento del $1 \%$ de la tasa de crecimiento de la industria del agua podría crear hasta 20.000 puestos de trabaj $0^{4}$.

4. La disminución de la oposición pública a la reutilización en general y a la reutilización indirecta como agua potable. La creciente confianza y buen resultado de las tecnologías emergentes como la ultrafiltración, la ósmosis inversa, los biorreactores de membrana y la desinfección ultravioleta han minimizado los posibles problemas de salud pública relacionados con la reutilización

4 Sanz \& Gawlik, 2014
En el gráfico 3 se muestra el crecimiento previsible de la capacidad instalada para los distintos grados de tratamiento a los que se puede someter el agua residual antes de su reutilización. Como se puede apreciar, el tipo de tratamiento que más crece es el terciario y avanzado.

Es interesante hacer mención del tratamiento que denominamos avanzado o emergente, dada su reciente aplicación, y que está basado en tratamiento del efluente secundario con membranas y desinfección adicional. Este tratamiento combina en serie el proceso de ultrafiltración (UF) o microfiltración (MF), seguido de ósmosis inversa (OI) y de desinfección mediante radiación ultravioleta (UV) u otro proceso de oxidación avanzada. Este triple tratamiento (UF/MF+OI+UV) obtiene un agua que cumple las normas más exigentes que se puedan establecer para el agua potable y, en consecuencia, permite su uso urbano o la inyección en acuíferos (o mezcla en embalses) para la reutilización potable indirecta.

Este tratamiento emergente se está empleando ya en varios países, por ej emplo:

- Desde 2008 en el Condado de Orange (Orange County Water District), en el Sur de California, hay en marcha un sistema de reposición de aguas subterráneas a partir de aguas residuales que se someten al sistema avanzado de purificación de agua. Son inyectadas en el acuífero de donde se extrae el agua potable, como barrera de intrusión marina para su reutilización potable. El proceso de purificación produce agua de alta calidad que supera los estándares del estado de California y los estándares federales de agua potable en USA. Se emplea para producir $260.000 \mathrm{~m}^{3} /$ día de agua. Está en funcionamiento desde enero de 2008, y desde entonces en expansión hasta 2015. El sistema de tratamiento puede producir $378.000 \mathrm{~m}^{3} /$ día, y se proyecta una capacidad última de $492.000 \mathrm{~m}^{3} /$ día. Se estima que este proceso contribuye con al rededor del $25 \%$ del suministro de agua dul- 
Gráfico 4. Evolución prevista para la capacidad instalada de tratamientos terciarios y avanzados por regiones

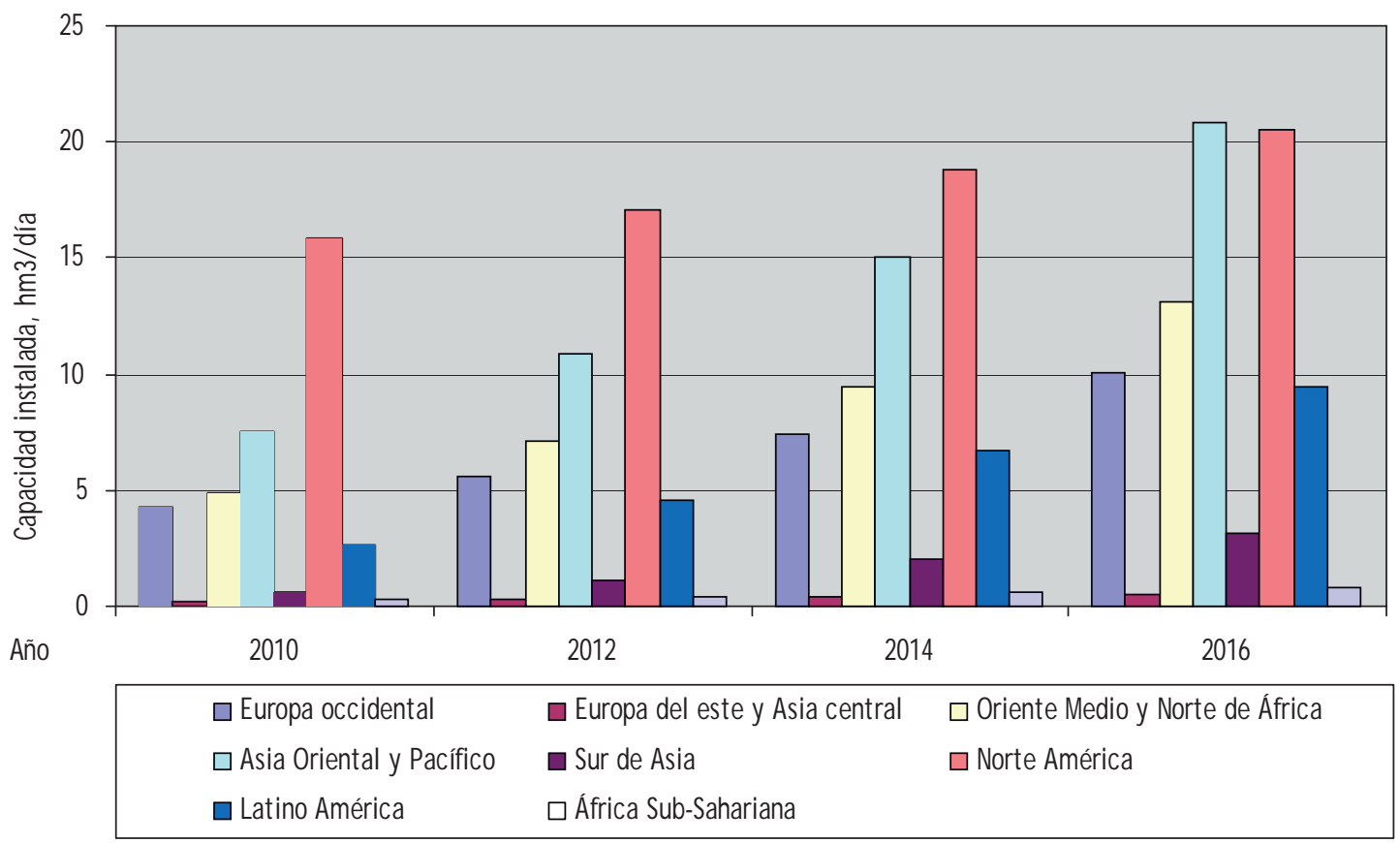

Fuente: elaboración de datos de Intelligence, 2009.

ce en la cuenca y disminuye la dependencia de agua importada costosa desde el norte de California y el río Colorado.

- En Singapur, la agencia nacional del agua introdujo en 2003 el proyecto NEWater (Singapore Water Reclamation), un proceso de barreras múltiples estrictas que supera los estándares de agua potable. Cuenta actualmente con cinco plantas con tratamiento avanzado con una capacidad total combinada de 462.000 $\mathrm{m}^{3} /$ día de agua regenerada. Parte de este agua se mezcla en los embalses con el agua que se emplea como agua potable. NEWater puede satisfacer actualmente el $30 \%$ de la demanda total de agua de Singapur, y se prevé que pueda satisfacer hasta el $55 \%$ de su demanda en 2060.

- En Perth, Australia, un primer proyecto de reposición de aguas subterráneas a gran escala comenzará a funcionar a finales de 2016 para asegurar el suministro de agua potable de la ciudad contra la disminución de la precipitación provocada por el cambio climático. El tratamiento incorpora la triple barrera de membranas y desinfección. El agua tratada recarga un acuífero profundo que se usará algunos años más tarde. La capacidad inicial es de catorce mil millones de litros/año, por lo que se convertirá en la próxima fuente de agua independiente del clima (ciclo del agua) para la creciente población de Perth, que también cuenta con dos plantas desaladoras de agua marina a gran escala. Este proyecto podrá suministrar hasta el 20\%de las necesidades de agua potable a más de dos millones de personas.

Mención aparte merece el caso de Namibia, donde, en la ciudad de Windhoek se cuenta con la primera, y de más larga duración, instalación de reutilización de agua potable directa. Lleva en funcionamiento desde 1968 y actualmente proporciona cerca del $35 \%$ del suministro total de agua potable para la ciudad. Windhoek tiene una población en rápido crecimiento de 325.000 personas en uno de los países más áridos de África del Sur. La planta de recuperación de Goreangab se ha convertido en una fuente de orgullo para una ciudad que sigue a la cabeza del mundo en la recuperación directa. En los más de cuarenta y cinco años de operación, el agua siempre ha cumplido con las normas de calidad, permitiendo proteger la salud de los consumidores. En este caso el tratamiento no incluye membranas, aunque está prevista una nueva planta incorporando la tecnología de biorreactores de membranas.

En España tenemos ejemplos de tratamiento avanzado en las depuradoras de Benidorm y de Rincón de León, en Alicante, cuyo efluente secundario se somete a UF + OI + UV y el agua regenerada se aplica fundamentalmente a regadío. En el caso de Benidorm permite permutar agua residual por agua dulce de acuífero (veremos con detalle este caso en el apartado de ejemplos del presente estudio).

Teniendo en cuenta cada uno de los posibles tratamientos de regeneración, la capacidad instalada de tratamientos para la reutilización de aguas residuales crecerá a escala mundial hasta alcanzar en 2016 los $130 \mathrm{hm}^{3} /$ día.

En el gráfico 4 se muestra la distribución de nuevos tratamientos terciarios, excluidos los emergentes, por regiones.

Como se puede observar, Norte América y Asia Oriental son las regiones con mayor previsión de crecimiento para este tipo de tratamientos.

Los cinco países en los que se prevé un mayor crecimiento en la construcción de tratamientos terciarios 0 avanzados para las aguas residuales urbanas son, por este orden, China, Estados Unidos, España, México y Australia. En la tabla 2 se indican los datos para estos países.

Podemos apreciar que España ocupa un lugar muy relevante en estas previsiones de crecimiento y es, sin duda, el país de 


\section{Daniel Prats-Rico}

Tabla 2. Países en los que se prevé un mayor crecimiento de la capacidad instalada de nuevos tratamientos terciarios 0 avanzados

\begin{tabular}{|l|c|c|c|c|}
\hline \multicolumn{1}{|c|}{ País } & $\begin{array}{c}\text { Millones } \\
\text { de habitantes } \\
(2008)\end{array}$ & $\begin{array}{c}\text { Caudal de aguas residuales } \\
\text { tratadas (hm³/ día) }\end{array}$ & $\begin{array}{c}\text { Porcentaje } \\
\text { de reutilización } \\
(2008)\end{array}$ & $\begin{array}{c}\text { Incremento previsto entre 2008 y 2016 } \\
\text { de la capacidad instalada de tratamientos } \\
\text { terciarios 0 avanzados (hm }{ }^{3} / \text { día) }\end{array}$ \\
\hline China & $1,338,0$ & 69,7 & 14 & 10,4 \\
\hline Estados Unidos & 307,0 & $1.192,2$ & 14 & 5,3 \\
\hline España & 40,5 & 11,6 & 11 & 3,5 \\
\hline México & 111,0 & 21,0 & 4 & 3,4 \\
\hline Australia & 21,3 & 5,4 & 15 & 2,4 \\
\hline
\end{tabular}

Fuente: elaborada con datos de Intelligence, 2009.

Gráfico 5. Potencial de reutilización en la UE

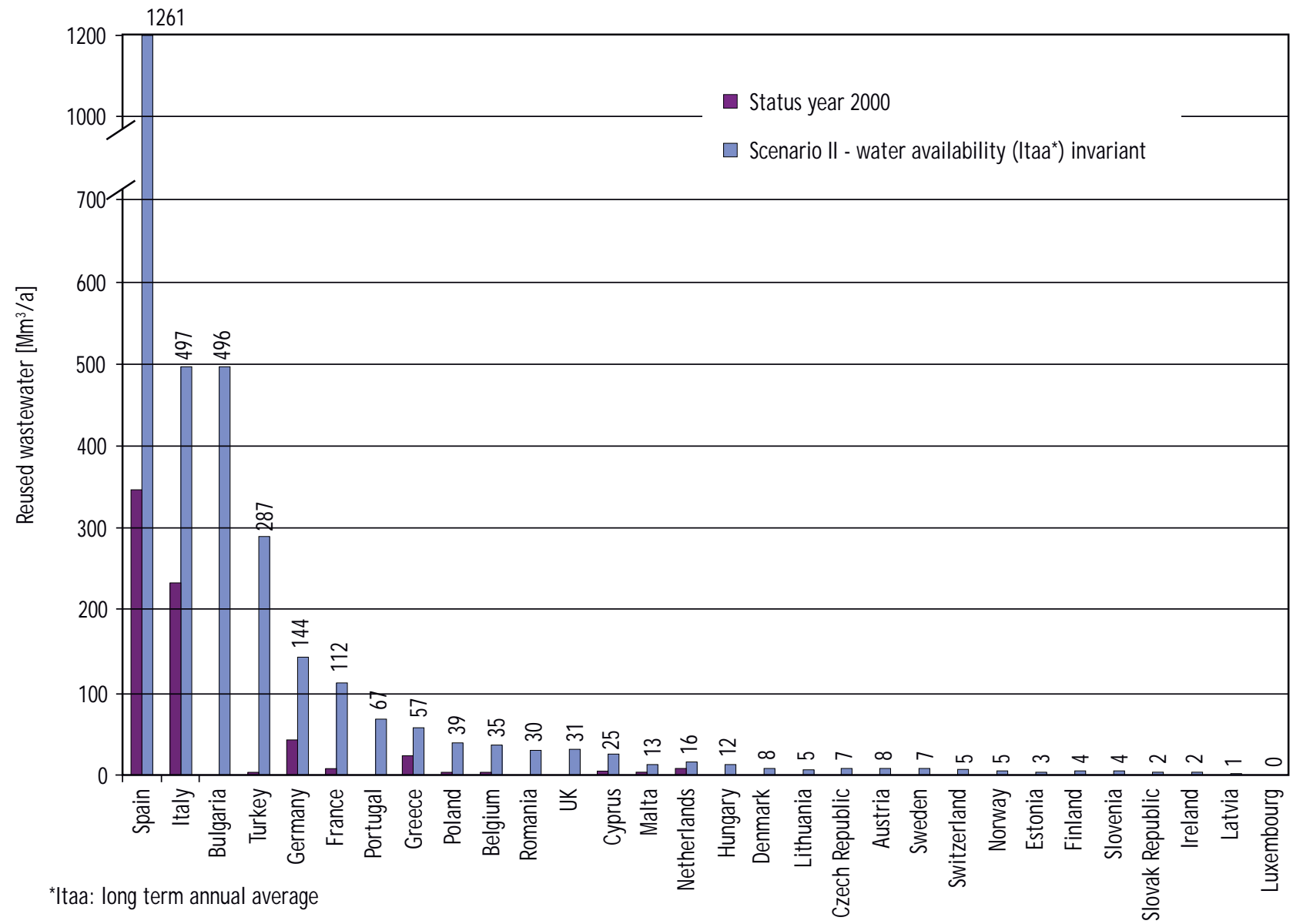

Fuente: Raso, 2013.

la UE con mayor capacidad de reutilización de aguas residuales. Raso ${ }^{5}$ realizó una proyección para los países de la UE hasta 2025 mostrando que España es el país que presenta un mayor potencial de crecimiento para la reutilización, que puede alcanzar 1.200 $\mathrm{Mm}^{3} /$ año. El resto de países con mayor potencial son Italia y Bulgaria con $500 \mathrm{Mm}^{3}$ / año, Turquía $287 \mathrm{Mm}^{3} /$ año, Alemania con 144 $\mathrm{Mm}^{3} /$ año y Francia con $112 \mathrm{Mm}^{3} /$ año. El resto de países tienen una previsión de reutilización inferior a $100 \mathrm{Mm}^{3}$ /año. En el gráfico 5 se muestra la capacidad de reutilización actual y la proyección para 2015.

\footnotetext{
5 Raso, 2013
}

\section{Biblografía}

Fluet, M. J., Vescovi, L., y Bokoye, A. I. 2009: The United Nations World Water Development Report 3: Water and Climate Change (Citizen Mobilization, a Source of Solutions). Paris, UNESCO.

Intelligence Global Water. 2009: Municipal Water Reuse Markets 2010. Media Analytics Ltd.

Jiménez, B. y Asano, T. 2008: Water Reuse: An International Survey of Current Practice. Issues and Needs. London, IWA Pub. DOI 10.2166/9781780401881.

Levine, Audrey D., Leverenz, Harold L. y Asano, T. 2010: "Water Reclamation and Reuse", in Water and Health [Ed. Willie O.K. Grabow], 
in Encyclopedia of Life Support Systems (EOLSS). Developed under the Auspices of the UNESCO, Oxford, Eolss Publishers.

Municipal Water Reuse Markets 2010. 2009: Analysis, forecasts and inventory. Oxford, Global Water Intelligence-Media Analytics Ltd.

National Research Council. Committee on the Assessment of Water Reuse as an Approach for Meeting Future Water Supply Needs. 2012: Water Reuse: Potential for Expanding the Nation's Water Supply Through Reuse of Municipal Wastewater. National Academies Press.

Rajendra, P. y Bhattarai, P. E. 2015: Water Reuse in Texas. Past, Present and Future. Twenty-ninth Surface Water Quality Monitoring: Annual Workshop. Camp Allen-Navasota, Texas.

Raso, J. 2013: Updated report on wastewater reuse in the European Union. Brussels, European Commission.
Sanz, L. A. y Gawlik, B. M. 2014: Water Reuse in Europe-Relevant guidelines, needs for and barriers to innovation. Brussels, J oint Research Centre.

Sato, T., Qadir, M., Yamamoto, S., Endo, T. y Zahoor, A. 2013: "Global, regional, and country level need for data on wastewater generation, treatment, and use", en Agricultural Water Management, 130, 1-13. http:// dx. doi. org/ 10.1016/ j. agwat. 2013.08.007

Schertenleib, M. H. y Egli-Brož, H. 2011: Globale Klimatologie: Meteorologie, Wetterinformation und Klimatologie. Compendio Bildungsmedien AG.

Werner, B. y Collins, R. 2012: Towards efficient use of water resources in Europe. Copenhagen, European Environment Agency.

World Water Development Report, 2015: Water for a Sustainable World. Paris, UNESCO. 